\title{
Thyroid autoimmunity and IVF/ICSI outcomes in euthyroid women: a systematic review and meta-analysis
}

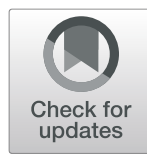

\author{
Ashley Venables', Wilbert Wong ${ }^{2}$, Mandy Way ${ }^{3}$ and Hayden Anthony Homer ${ }^{4,5,6^{*}}$ (D)
}

\begin{abstract}
Background: Thyroid autoimmunity (TAI) - the presence of anti-thyroid peroxidase and/or anti-thyroglobulin antibodies - affects $8-14 \%$ of reproductively-aged women. It is hotly debated whether TAl adversely affects IVF/ICSI outcomes. This systematic review and meta-analysis evaluated the relationship between thyroid autoimmunity (TAI) and IVF/ICSI outcomes, both overall and amongst euthyroid women of known age using strict criteria for grouping pregnancy outcomes.
\end{abstract}

Methods: The review was registered with PROSPERO: CRD42019120947. Searches were undertaken in MEDLINE, EMBASE, Web of Science and Cochrane Database from Inception-March 2020. Primary outcomes were clinical pregnancy rate, clinical miscarriage rate, biochemical pregnancy loss, livebirth rate per-cycle and live birth rate per clinical pregnancy $(C P)$.

Results: 14 studies were included in the meta-analysis. Compared with women who tested negative for thyroid autoantibodies (TAl-), there was no significant difference in clinical pregnancy rate overall (OR 0.86; $95 \% \mathrm{Cl}[0.70$, $1.05] ; P=0.14 ; 11$ studies; $\left.I^{2}=29.0 \%\right)$, or in euthyroid women (OR 0.88; 95\%Cl $[0.69,1.12] ; P=0.29 ; 10$ studies; $I^{2}=$ $32.0 \%)$. There was also no significant difference in clinical miscarriage rate overall $(\mathrm{OR} 1.04 ; 95 \% \mathrm{Cl}[0.52,2.07] ; P=$ $0.908 ; 8$ studies; $I^{2}=53 \%$ ), or in euthyroid women (OR 1.18; 95\%Cl $[0.52,2.64] ; P=0.69 ; 7$ studies; $\left.I^{2}=54 \%\right)$. There was no significant difference in biochemical pregnancy loss (OR 1.14; $95 \% \mathrm{Cl}[0.48,2.72] ; P=0.769 ; 4$ studies; $12=$ $0.0 \%)$, live birth rate per cycle (OR $\left.0.84 ; 95 \% \mathrm{Cl}[0.67,1.06] ; P=0.145 ; I^{2}=1.7 \%\right)$, live birth rate per clinical pregnancy (OR 0.67; 95\%Cl $[0.28,1.60] ; P=0.369 ; I^{2}=69.2 \%$ ), both overall and in euthyroid women as all studies included consisted of euthyroid women only. There was also no significant difference in number of embryos transferred, number of oocytes retrieved, mean maternal age or TSH levels overall or in euthyroid women.

Conclusion: The findings of the present study suggest that thyroid autoimmunity has no effect on pregnancy outcomes in euthyroid women alone, or in euthyroid women and women with subclinical hypothyroidism.

Keywords: Thyroid autoimmunity, TAI, IVF, ICSI, ART, TSH, Pregnancy outcomes

\footnotetext{
* Correspondence: h.homer@uq.edu.au

${ }^{4}$ Christopher Chen Oocyte Biology Research Laboratory, UQ Centre for

Clinical Research, The University of Queensland, Brisbane, Queensland,

Australia

${ }^{5}$ Reproductive Endocrinology and Infertility Clinic, Royal Brisbane \& Women's

Hospital, Brisbane, Queensland, Australia

Full list of author information is available at the end of the article
}

\section{$\triangle B M C$}

(c) The Author(s). 2020 Open Access This article is licensed under a Creative Commons Attribution 4.0 International License, which permits use, sharing, adaptation, distribution and reproduction in any medium or format, as long as you give appropriate credit to the original author(s) and the source, provide a link to the Creative Commons licence, and indicate if changes were made. The images or other third party material in this article are included in the article's Creative Commons licence, unless indicated otherwise in a credit line to the material. If material is not included in the article's Creative Commons licence and your intended use is not permitted by statutory regulation or exceeds the permitted use, you will need to obtain permission directly from the copyright holder. To view a copy of this licence, visit http://creativecommons.org/licenses/by/4.0/ The Creative Commons Public Domain Dedication waiver (http://creativecommons.org/publicdomain/zero/1.0/) applies to the data made available in this article, unless otherwise stated in a credit line to the data. 


\section{Background}

Thyroid autoimmunity (TAI) is defined as the presence of antithyroid antibodies - anti-thyroid peroxidase (TPO-Ab) and/or anti-thyroglobulin (TG-Ab) antibodies - and is the most common autoimmune condition in women of reproductive age, with a prevalence of $8-14 \%$ $[1,2]$. Significantly, TAI has an even higher prevalence of up to $20 \%$ in infertile women [3]. Even when thyroid function remains normal, TAI has been associated with adverse pregnancy outcomes including spontaneous miscarriage and preterm birth $[1,4,5]$. Ovarian stimulation used during IVF/ICSI places additional demands on thyroid function [6, 7], which may potentially expose women with TAI (TAI+) to additional risks. Consequently, there has been intense interest in whether TAI compromises outcomes during IVF/ICSI treatment.

Multiple systematic reviews have investigated the relationship between TAI and IVF/ICSI outcomes, however there is substantial discrepancy between findings. Toulis et al. (2010) [8], found that sub-fertile TAI+ women undergoing IVF were at significantly greater risk of miscarriage than women without TAI (TAI-) but did not identify differences in either clinical pregnancy rates (CPR) or delivery rates. Thangaratinam et al. (2011) [9] also found a significantly higher miscarriage rate (MR) amongst TAI+ women. TAI+ women were found to have a greater risk of spontaneous miscarriage in addition to lower live birth rates (LBR) with no reductions in either CPR or implantation rates (IR) [6]. On the contrary, He et al. (2016) [10] found no association between TAI positivity and clinical pregnancy, miscarriage rate or delivery rate. Likewise, Leiva et al. (2017) [11] found no association between TAI and increased risk of pregnancy loss.

For a number of reasons, it is important that this topic be revisited. Firstly, earlier meta-analyses combined outcomes that were defined differently in different studies. For instance, some papers considered miscarriage to include both clinical and biochemical pregnancy losses (BPL) (e.g. Muller et al. 1999 [12], Poppe et al. 2003 [13]) whereas others included only clinical losses (e.g. Chai et al. 2014 [14]; Lucaszuk et al. 2015 [15]). In the most recent comprehensive meta-analysis on the effect of TAI on IVF outcomes, all miscarriage types, whether biochemical or clinical were pooled into a single analysis [6]. For uniformity, it is critically important to analyse each miscarriage type separately since biochemical and clinical losses have very different frequencies and aetiologies [16]. Biochemical pregnancy loss in IVF is estimated to have an incidence of up to $22 \%$ [17], compared to clinical miscarriage which has an incidence of $\sim 15 \%$ $[18,19]$. A number of mechanisms have been proposed by which, TAI might increase miscarriage risk including detrimental effects on the endometrium, embryo toxicity, general immune disturbance and compromised ability of thyroid function to satisfy increased demands of pregnancy [2]. It is conceivable that very early postimplantation pregnancy stages might have a different vulnerability to these threats than later stages. To explore whether stage-specific vulnerability to TAI might exist, we not only analysed outcomes for all miscarriages, but also differentiated between biochemical and clinical losses whenever possible. For LBR analyses, some papers calculated LBR on a per-cycle basis whereas others calculated on a per-clinical pregnancy $(\mathrm{CP})$ basis; again, these were combined into a single analysis [6]. As with MR outcomes, it is important that like-for-like livebirth outcomes are compared. Notably, similar concerns also apply to the two meta-analyses on this topic performed prior to $2016[8,9]$.

A second critically important consideration is that female age was not reported in 3 of the 12 studies included in the most recent comprehensive meta-analysis [6]. This is pivotal since arguably the most important determinant of pregnancy outcome is female age due to the effects of ageing on oocyte quality, which ultimately impacts aneuploidy rates [20]. Moreover, when age was analysed for the 9 papers in which maternal age was known, Busnelli et al. (2016) [6] found that TAI+ women were significantly older than TAI- women. Based on these analyses, therefore, it is very unclear to what extent the older age of the TAI+ cohort contributed to the adverse reproductive outcomes that were observed.

Thirdly, since the last meta-analysis, at least 4 further studies analysing women of known ages have been published [21-24]. Busnelli et al. (2016) [6] included a total of $428 \mathrm{TAI}+$ women of known ages. These 3 newer studies [21-24] contain an additional 390 TAI+ women.

Fourthly, half of the 12 studies included in Busnelli et al. (2016) [6] were published prior to 2010. In more recent years, guidelines from the ASRM and the American Thyroid Association [25] suggest that a lower thyroid stimulating hormone (TSH) cut-off $(2.5 \mathrm{mIU} / \mathrm{mL})$ should be used for initiating levothyroxine treatment in TAI+ women. Newer studies may therefore include women with more intact thyroid function whereas older studies may have included patients with more severe thyroid imbalances, which may have increased the tendency to adverse outcomes in earlier meta-analyses. Indeed, two of the studies in the recent meta-analysis [12, 26] included women with overtly abnormal TSH levels. It is notable in this regard that the 2011 meta-analysis identified an increased odds ratio (OR) for miscarriage of 3.15 amongst TAI+ women [9] whereas in Busnelli et al. (2016) [6], the OR had declined to less than half this at 1.44 [6]. Furthermore, in He et al. (2016) [10] and Leiva et al. (2017) [11], no significant relationship was found between TAI and IVF outcomes. While $\mathrm{He}$ et al. 
(2016) [10] found no association between TAI positivity and clinical pregnancy, miscarriage rate or delivery rate in euthyroid women, they did find that women with subclinical hypothyroidism $(\mathrm{SCH})$ and TAI were at greater risk of miscarriage and had a lower delivery rate than TAI- women, indicating that the extent of associated thyroid dysfunction, rather than TAI per se, could account in some part for adverse outcomes reported in previous studies.

Here we undertook an updated systematic review and meta-analysis of the overall relationship between TAI and IVF/ICSI outcomes as has been done previously, as well as separate analyses of euthyroid women of known female age using strict criteria for defining pregnancy outcomes.

\section{Materials and methods}

\section{Protocol and registration}

PRISMA guidelines were followed and the review was registered with PROSPERO (Registration no. CRD42019120947).

\section{Search strategy}

Literature searches were conducted via MEDLINE, EMBASE, The Cochrane Library (Cochrane Database of Systematic Reviews, Cochrane Central Register of Controlled Trials (CENTRAL), Cochrane Methodology Register), and Web of Science (Science and Social Science Citation Index) from inception to April 2019. Searches were rerun in March 2020 prior to publication. The Medline search utilised a combination of text and medical subject headings (MeSH): ("Thyroid Gland"[Mesh] AND "Autoimmunity"[Mesh]) OR "thyroid autoimmunity" OR "thyroid autoantibody" OR "thyroid autoantibodies" OR antithyroid peroxidase OR "antithyroglobulin antibody", "Fertilization in Vitro"[Mesh] OR IVF OR "invitro fertilization" OR "in vitro fertilization" OR "in-vitro fertilization" OR "invitro fertilization" OR "invitro fertilisation" OR "in vitro fertilisation" OR "invitro fertilisation" OR "invitro fertilisation" OR "Sperm Injections, Intracytoplasmic"[Mesh] OR "intracytoplasmic sperm injection" OR ICSI OR ART [tiab] OR "reproductive techniques, assisted"[MeSH Terms] OR "assisted reproductive technology". Search terms were set by the authors and were adapted for use with other databases. No language restrictions were implemented. Peer reviewed case control and cohort studies were eligible for inclusion.

\section{Study selection}

Title and abstract screening were performed independently by two authors (A.V. and W.W.) to eliminate studies deemed irrelevant. The full text versions of relevant studies were retrieved and assessed for eligibility by the same author. Any uncertainty was resolved through discussion with a third author. Studies were excluded if: (i) women had overt thyroid dysfunction, (ii) maternal age was not reported, (iii) women were being treated with levothyroxine, (iv) ART techniques aside from IVF/ICSI were used. The quality of the studies was assessed using the Newcastle Ottawa scale [27].

\section{Data extraction and analysis}

Two authors extracted data independently. Study year, setting, design, thyroid antibody assays used and cut-off values, number of TAI+ and TAI- patients, number of IVF/ ICSI cycles performed, type of ART procedure performed and key outcomes of the study were recorded. Extracted data was compared to ensure accuracy, and discrepancies were corrected upon referral back to the original paper.

The primary outcomes measured were $\mathrm{CPR}, \mathrm{MR}$, and LBR as; (i) CP: the presence of a gestational sac on ultrasound scan, CPR: the proportion of IVF/ICSI cycles resulting in a $\mathrm{CP}$; (ii) $\mathrm{MR}$ : the proportion of pregnancies that miscarried/did not result in a delivery or live birth, CMR: the proportion of clinical pregnancies that miscarried/did not result in a delivery or live birth, BPL: the proportion of biochemical pregnancies that were lost prior to the CP stage (iii) LBR: the proportion of cycles of IVF/ICSI that resulted in the birth of at least one live baby, calculated per cycle and per CP. In cases where outcomes of interest were not reported by the study but could be determined from the raw data, outcomes were calculated manually. Secondary outcomes such as overall MR (including all definitions of MR: BPR + CMR + CMR alone), maternal age, number of oocytes retrieved (NOR), and numbers of embryos transferred (ET) were also recorded.

\section{Statistical method}

Data were analysed in STATA 15 [28]. Pooled effect sizes were estimated by applying random effects metaanalysis. The $\mathrm{I}^{2}$ statistic was used as a measure of heterogeneity between studies. An $\mathrm{I}^{2}$ value of $0 \%$ indicates no heterogeneity whereas values of 25,50 and $75 \%$ reflect low, moderate and high heterogeneity, respectively [29]. Egger's weighted regression test was used to assess publication bias where more than 10 studies were included in the analysis. Sensitivity analyses were performed where moderate levels of heterogeneity were detected.

\section{Results}

Results of literature search and description of included studies

Systematic searches of the PubMed, Embase, Web of Science and Cochrane Library databases identified 491 
citations, with 332 of these being non-duplicates (Fig. 1). 274 articles were excluded based on title and abstract screening. The full texts of 58 articles were retrieved. An additional 45 articles were excluded (Fig. 1) on the following basis: 5 articles did not report maternal age, 7 articles did not include either a TAI+ group or a TAIcontrol group, 3 studies included patients on levothyroxine, 3 were review articles, 3 did not report patient outcomes, 19 were conference abstracts, 1 did not use IVF/ ICSI, 2 were excluded due to patient overlap and 2 studies were excluded during data analysis due to discrepancies in results (additional file 1). One more study was added in March 2020 when searches were rerun. Details of the 14 studies that were included in the final analyses are presented in Table 1.

\section{Outcomes}

\section{CPR}

Overall CPR was reported by 11 studies. No significant difference in CPR was observed between TAI+ and TAIwomen (OR 0.86 ; $95 \%$ CI $[0.70,1.05] ; P=0.14 ; 11$ studies; $\left.\mathrm{I}^{2}=29.0 \%\right)($ Fig. 2a). CPR in euthyroid women was reported by 10 studies. No significant difference in CPR was observed between TAI+ and TAI- women (OR 0.88; $95 \%$ CI $\quad[0.69,1.12] ; \quad P=0.29 ; \quad 10 \quad$ studies; $\mathrm{I}^{2}=$ 32.0\%)(Fig. 2b).

\section{$M R$}

Overall CMR was reported by 8 studies. No significant difference was observed in CMR between $\mathrm{TAI}+$ and TAI- women (OR 1.04; 95\%CI [0.52, 2.07]; $P=0.908 ; 8$ studies; $\mathrm{I}^{2}=53 \%$ ) (Fig. 3a). CMR in euthyroid women was reported by 7 studies. No significant difference was observed in CMR between TAI+ and TAI- women (OR 1.18; $95 \%$ CI $[0.52,2.64]$; $P=0.69 ; 7$ studies; $\mathrm{I}^{2}=54 \%$ ) (Fig. 3b). BPL was reported by 4 studies, all of which only included euthyroid women (Fig. 4). No significant difference was observed in BPL between TAI+ and TAIwomen (OR 1.14; 95\%CI $[0.48,2.72] ; P=0.769 ; 4$ studies; $\mathrm{I} 2=0.0 \%)$. 11 studies reported overall MR using a variety of definitions. No significant difference in $M R$ based on all definitions combined was observed (OR 1.33 ; $95 \%$ CI $[0.83-2.15] ; \quad P=0.23 ; 11$ studies; $\mathrm{I}^{2}=$ 47\%)(Fig. 5a). 8 studies reported MR using a variety of

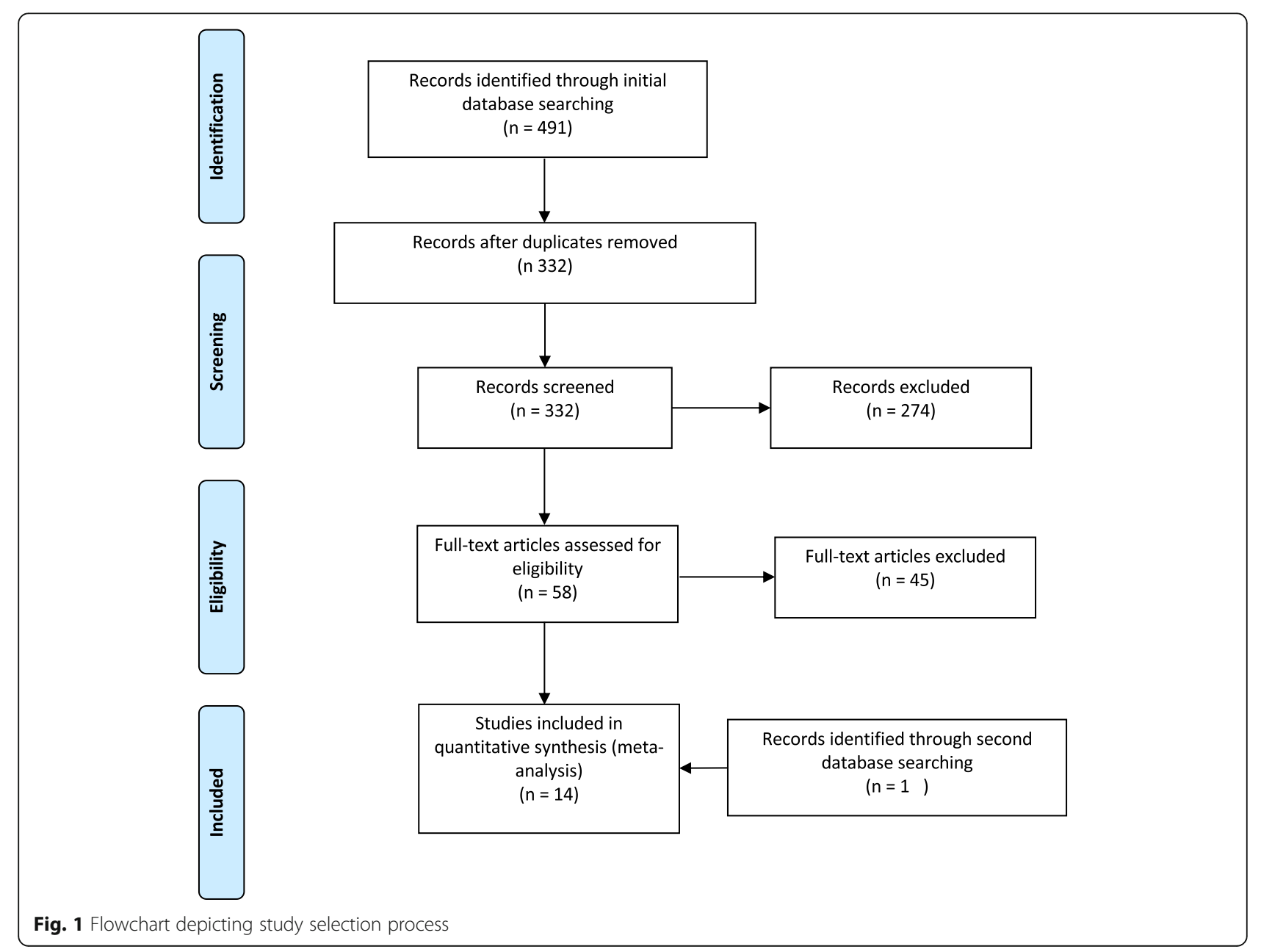









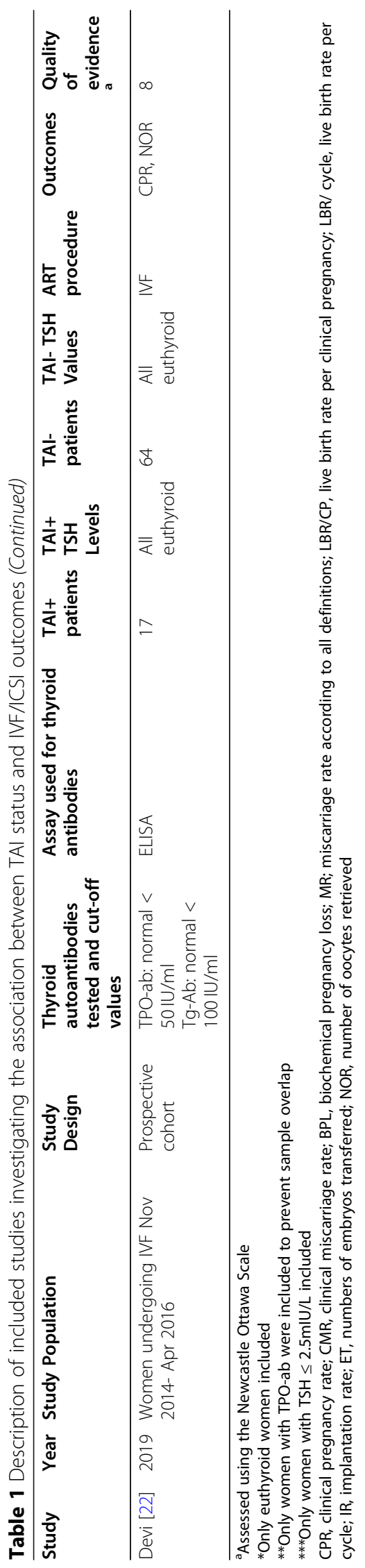




\begin{tabular}{|c|c|c|c|c|c|c|c|c|c|c|}
\hline \multirow[t]{15}{*}{ a } & Study or Subgroup & \multirow{2}{*}{$\begin{array}{r}\begin{array}{r}\text { TAl+ } \\
\text { Events }\end{array} \\
16\end{array}$} & \multirow{2}{*}{$\begin{array}{r}\text { Total } \\
32\end{array}$} & $\begin{array}{l}\text { TAl- } \\
\text { Events }\end{array}$ & \multirow{2}{*}{$\begin{array}{r}\text { Total } \\
202\end{array}$} & \multirow{2}{*}{$\begin{aligned} \text { Weight } \\
6.0 \%\end{aligned}$} & $\begin{array}{l}\text { Odds Ratio } \\
\text { M-H, Random, } 95 \% \text { Cl Year }\end{array}$ & \multicolumn{3}{|c|}{$\begin{array}{c}\text { Odds Ratio } \\
\text { M-H, Random, } 95 \% \mathrm{Cl}\end{array}$} \\
\hline & Poppe 2003 & & & 77 & & & $1.62[0.77,3.43] 2003$ & & & \\
\hline & Kilic 2008 & 7 & 23 & 13 & 31 & $2.9 \%$ & $0.61[0.19,1.89] 2008$ & & & \\
\hline & Revelli 2009 & 4 & 52 & 66 & 200 & $3.3 \%$ & $0.17[0.06,0.49] 2009$ & & & \\
\hline & Karacan 2013 & 12 & 34 & 89 & 219 & $5.9 \%$ & $0.80[0.38,1.69] 2013$ & & & \\
\hline & Chai 2014 & 40 & 89 & 192 & 419 & $12.3 \%$ & $0.97[0.61,1.53] 2014$ & & - & \\
\hline & Tan 2014 & 45 & 110 & 301 & 725 & $14.2 \%$ & $0.98[0.65,1.47] 2014$ & & & \\
\hline & Lukaszuk 2015 & 50 & 114 & 235 & 495 & $14.1 \%$ & $0.86[0.57,1.30] 2015$ & & 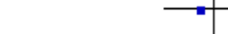 & \\
\hline & Sakar 2016 & 7 & 32 & 26 & 126 & $4.0 \%$ & $1.08[0.42,2.76] 2016$ & & & \\
\hline & Zhuang 2017 & 57 & 127 & 305 & 662 & $15.3 \%$ & $0.95[0.65,1.40] 2017$ & & & \\
\hline & Chen 2017 & 116 & 214 & 515 & 844 & $19.3 \%$ & $0.76[0.56,1.02] 2017$ & & & \\
\hline & Devi 2019 & 5 & 16 & 24 & 61 & $2.7 \%$ & $0.70[0.22,2.27] 2019$ & & & \\
\hline & Total $(95 \% \mathrm{Cl})$ & & 843 & & 3984 & $100.0 \%$ & $0.86[0.70,1.05]$ & & & \\
\hline & Total events & 359 & & 1843 & & & & & & \\
\hline & $\begin{array}{l}\text { Heterogeneity: } \mathrm{Tau}^{2}= \\
\text { Test for overall effect: }\end{array}$ & $\begin{array}{l}0.03 ; \mathrm{Chi}^{2} \\
\mathrm{Z}=1.49(\mathrm{P}\end{array}$ & $\begin{array}{l}=14.18 \\
=0.14\end{array}$ & 4) $d f=10$ & $(P=0$. & $16) ; I^{2}=29$ & & 0.05 & $\begin{array}{c}0.2 \\
\text { Decreased in TAl+ }\end{array}$ & $\begin{array}{cc}5 & 20 \\
\text { Increaased in TAl+ } & \end{array}$ \\
\hline b & Study or Subgroup & $\begin{array}{c}\text { TAl+ } \\
\text { Events }\end{array}$ & Total & $\begin{array}{l}\text { TAI- } \\
\text { Events }\end{array}$ & Total & Weight & $\begin{array}{l}\text { Odds Ratio } \\
\text { M-H, Random, } 95 \% \text { Cl Year }\end{array}$ & & $\begin{array}{r}\text { Odds } \mathrm{F} \\
\text { M-H, Rando }\end{array}$ & $\begin{array}{l}\text { Ratio } \\
\text { m, } 95 \% \mathrm{Cl}\end{array}$ \\
\hline & Poppe 2003 & 16 & 32 & 77 & 202 & $7.8 \%$ & $1.62[0.77,3.43] 2003$ & & & \\
\hline & Kilic 2008 & 7 & 23 & 13 & 31 & $3.9 \%$ & $0.61[0.19,1.89] 2008$ & & & \\
\hline & Revelli 2009 & 4 & 52 & 66 & 200 & $4.4 \%$ & $0.17[0.06,0.49] 2009$ & & & \\
\hline & Karacan 2013 & 12 & 34 & 89 & 219 & $7.7 \%$ & $0.80[0.38,1.69] 2013$ & & & \\
\hline & Tan 2014 & 45 & 110 & 301 & 725 & $17.0 \%$ & $0.98[0.65,1.47] 2014$ & & & \\
\hline & Chai 2014 & 40 & 89 & 192 & 419 & $15.0 \%$ & $0.97[0.61,1.53] 2014$ & & & \\
\hline & Lukaszuk 2015 & 50 & 114 & 235 & 495 & $17.0 \%$ & $0.86[0.57,1.30] 2015$ & & $=$ & - \\
\hline & Sakar 2016 & 7 & 32 & 26 & 126 & $5.4 \%$ & $1.08[0.42,2.76] 2016$ & & & \\
\hline & Zhuang 2017 & 57 & 127 & 305 & 662 & $18.2 \%$ & $0.95[0.65,1.40] 2017$ & & & \\
\hline & Devi 2019 & 5 & 16 & 24 & 61 & $3.7 \%$ & $0.70[0.22,2.27] 2019$ & & & \\
\hline & Total $(95 \% \mathrm{Cl})$ & & 629 & & 3140 & $100.0 \%$ & $0.88[0.69,1.12]$ & & & \\
\hline & Total events & 243 & & 1328 & & & & & & \\
\hline & $\begin{array}{l}\text { Heterogeneity: } \mathrm{Tau}^{2}= \\
\text { Test for overall effect: }\end{array}$ & $\begin{array}{l}0.04 ; \mathrm{Chi}^{2} \\
\mathrm{Z}=1.06(P\end{array}$ & $\begin{array}{l}=13.27 \\
=0.29\end{array}$ & 7) $d f=9(F$ & $P=0.1$ & $5) ; I^{2}=32 \%$ & & 0.05 & $\begin{array}{c}0.2 \\
\text { Decreased in TAl+ }\end{array}$ & $\begin{array}{cc}1 & + \\
\text { Increaased in TAl+ } & 20\end{array}$ \\
\hline & $\begin{array}{l}2 \text { a Overall association } \\
\text { between TAl+ and TAl } \\
\text { hood of clinical pregnar } \\
95 \% \mathrm{Cl}[0.69,1.12] ; \mathrm{P}=0\end{array}$ & $\begin{array}{l}\text { between } \\
\text { - women o } \\
\text { lcy in euth } \\
\text { 29; } 10 \text { stuc }\end{array}$ & $\begin{array}{l}\text { thyroid } \\
\text { overall ( } \\
\text { yyroid w } \\
\text { diec. }\left.\right|^{2}=\end{array}$ & $\begin{array}{l}\text { autoimm } \\
\text { (OR 0.86; } 9 \\
\text { vomen. Re } \\
-3200 \% \text { ) }\end{array}$ & $\begin{array}{l}\text { nunity a } \\
95 \% \text { Cl [ } \\
\text { esults sh }\end{array}$ & $\begin{array}{l}\text { nd likeliho } \\
\text { 0.70, 1.05]; } \\
\text { howed no }\end{array}$ & $\begin{array}{l}\text { ood of clinical pregnancy. Results } \\
\left.; P=0.14 ; 11 \text { studies; }\left.\right|^{2}=29.0 \%\right) \text {. } \\
\text { significant difference in clinical }\end{array}$ & s show & $\begin{array}{l}\text { red no significant diffe } \\
\text { ociation between thy } \\
\text { ancy rates in TAl+ an } \\
\text { ne; OR, odds ratio; Cl, }\end{array}$ & $\begin{array}{l}\text { rence in clinical pregnancy } \\
\text { oid autoimmunity and } \\
\text { TAl- euthyroid women (OR }\end{array}$ \\
\hline
\end{tabular}

definitions in euthyroid women. No significant difference in MR based on all definitions combined was observed (OR 1.26; 95\%CI [0.72-2.18]; $P=0.42 ; 8$ studies; $\left.\mathrm{I}^{2}=35 \%\right)$ (Fig. 5b).

\section{$L B R$}

LBR per CP was reported by 4 studies, all of which only included euthyroid women. No significant difference was observed in LBR between TAI+ and TAI- women (OR 0.67; 95\%CI $[0.28,1.60] ; P=0.369 ; \mathrm{I}^{2}=69.2 \%$ )(Fig. 6). LBR per cycle was reported by 5 studies and no significant difference was observed in LBR per cycle between TAI+ and TAI- women (OR 0.84; 95\%CI $[0.67,1.06]$; $P=0.145 ; \mathrm{I}^{2}=1.7 \%$ ) (Fig. 7).

\section{ET}

Overall mean number of embryos transferred was reported by 5 studies. No significant difference in mean number of transferred embryos was observed between TAI+ and TAI- women (SMD 0.02; 95\%CI [- 0.18, 0.22];
$P=0.849 ; \mathrm{I}^{2}=69 \%$ ) (Fig. 8a). Mean number of embryos transferred in euthyroid women was reported by 3 studies. No significant difference in mean numbers was observed between TAI+ and TAI- women (SMD 0.07; 95\%CI [- 0.13, 0.27]; $P=0.49 ; \mathrm{I}^{2}=0 \%$ ) (Fig. 8b).

\section{NOR}

Overall number of oocytes retrieved (NOR) was reported by 9 studies. No significant difference was observed between TAI+ and TAI- women (SMD -0.07; 95\%CI [$0.20,0.06$ ]; $P=0.27 ; 9$ studies; $\mathrm{I}^{2}=49 \%$ )(Fig. 9a). NOR in euthyroid women was reported by 7 studies. No significant difference was observed between TAI+ and TAIwomen (SMD $-0.05 ; 95 \%$ CI $[-0.25,0.16] ; P=0.67 ; 7$ studies; $\mathrm{I}^{2}=60 \%$ )(Fig. 9b).

\section{Age}

Age was reported by all 14 studies. One study reported age as the median (25th-75th) [15] and could not therefore be included in our analysis of mean ages. However, 


\begin{tabular}{|c|c|c|c|c|c|c|c|c|c|c|}
\hline$a$ & Study or Subgroup & $\begin{array}{c}\text { TAl+ } \\
\text { Events }\end{array}$ & Total & $\begin{array}{l}\text { TAI- } \\
\text { Events }\end{array}$ & Total & Weight & $\begin{array}{l}\text { Odds Ratio } \\
\text { M-H, Random, } 95 \% \mathrm{Cl} \text { Year }\end{array}$ & & $\begin{array}{r}\text { Odds } \mathrm{F} \\
\text { M-H, Rando }\end{array}$ & $\begin{array}{l}\text { Ratio } \\
\text { m, } 95 \% \mathrm{Cl}\end{array}$ \\
\hline & Poppe 2003 & 8 & 16 & 10 & 77 & $14.7 \%$ & $6.70[2.05,21.89] \quad 2003$ & & & $\rightarrow$ \\
\hline & Revelli 2009 & 1 & 4 & 8 & 66 & $6.3 \%$ & $2.42[0.22,26.13] \quad 2009$ & & & \\
\hline & Karacan 2013 & 1 & 12 & 8 & 89 & $7.2 \%$ & $0.92[0.10,8.08] 2013$ & & & \\
\hline & Chai 2014 & 8 & 40 & 37 & 192 & $18.5 \%$ & $1.05[0.45,2.46] 2014$ & & & \\
\hline & Tan 2014 & 2 & 45 & 29 & 301 & $11.9 \%$ & $0.44[0.10,1.89] 2014$ & & & \\
\hline & Lukaszuk 2015 & 3 & 50 & 29 & 235 & $14.2 \%$ & $0.45[0.13,1.55] 2015$ & & & \\
\hline & Sakar 2016 & 2 & 7 & 7 & 26 & $9.0 \%$ & $1.09[0.17,6.94] 2016$ & & & \\
\hline & Chen 2017 & 6 & 116 & 43 & 515 & $18.2 \%$ & $0.60[0.25,1.44] 2017$ & & & \\
\hline & Total $(95 \% \mathrm{Cl})$ & & 290 & & 1501 & $100.0 \%$ & $1.04[0.52,2.07]$ & & & \\
\hline & Total events & 31 & & 171 & & & & & & \\
\hline & $\begin{array}{l}\text { Heterogeneity: } \mathrm{Tau}^{2}= \\
\text { Test for overall effect: }\end{array}$ & $\begin{array}{l}0.48 ; \mathrm{Chi}^{2} \\
=0.12(\mathrm{~F}\end{array}$ & $\begin{array}{l}=14.75 \\
=0.91\end{array}$ & $\begin{array}{l}5, \mathrm{df}=7(\mathrm{~F} \\
\text { 1) }\end{array}$ & $P=0.0$ & $4) ;\left.\right|^{2}=53^{\circ}$ & & 0.05 & $\begin{array}{c}0.2 \\
\text { Decreased in TAl+ }\end{array}$ & $\begin{array}{c}5 \\
\text { Increaased in TAl+ }\end{array}$ \\
\hline b & Study or Subgroup & $\begin{array}{c}\text { TAl+ } \\
\text { Events }\end{array}$ & Total & $\begin{array}{c}\text { TAl- } \\
\text { Events }\end{array}$ & Total & Weight & $\begin{array}{l}\text { Odds Ratio } \\
\text { M-H, Random, } 95 \% \mathrm{Cl} \text { Year }\end{array}$ & & $\begin{array}{r}\text { Odds } \mathrm{F} \\
\text { M-H, Rando }\end{array}$ & $\begin{array}{l}\text { Ratio } \\
\text { m, } 95 \% \mathrm{Cl}\end{array}$ \\
\hline & Poppe 2003 & 8 & 16 & 10 & 77 & $17.7 \%$ & $6.70[2.05,21.89] 2003$ & & & $\rightarrow$ \\
\hline & Revelli 2009 & 1 & 4 & 8 & 66 & $8.2 \%$ & $2.42[0.22,26.13] 2009$ & & & \\
\hline & Karacan 2013 & 1 & 12 & 8 & 89 & $9.3 \%$ & $0.92[0.10,8.08] 2013$ & & & \\
\hline & Chai 2014 & 8 & 40 & 37 & 192 & $21.6 \%$ & $1.05[0.45,2.46] 2014$ & & & \\
\hline & Tan 2014 & 2 & 45 & 29 & 301 & $14.7 \%$ & $0.44[0.10,1.89] 2014$ & & & \\
\hline & Lukaszuk 2015 & 3 & 50 & 29 & 235 & $17.2 \%$ & $0.45[0.13,1.55] 2015$ & & & \\
\hline & Sakar 2016 & 2 & 7 & 7 & 26 & $11.4 \%$ & $1.09[0.17,6.94] 2016$ & & & \\
\hline & Total $(95 \% \mathrm{Cl})$ & & 174 & & 986 & $100.0 \%$ & $1.18[0.52,2.64]$ & & & \\
\hline & Total events & 25 & & 128 & & & & & & \\
\hline & $\begin{array}{l}\text { Heterogeneity: } \mathrm{Tau}^{2}= \\
\text { Test for overall effect: }\end{array}$ & $\begin{array}{l}\text {. } 60 ; \mathrm{Chi}^{2} \\
=0.39(\mathrm{~F}\end{array}$ & $\begin{array}{l}=13.03 \\
P=0.65\end{array}$ & $\begin{array}{l}3, \mathrm{df}=6(\mathrm{P} \\
\text { 9) }\end{array}$ & $P=0.0$ & $4) ;\left.\right|^{2}=54 \%$ & & 0.05 & $\begin{array}{c}0.2 \\
\text { Decreased in TAl+ }\end{array}$ & $\begin{array}{c}5 \\
\text { Increaased in TAl+ }\end{array}$ \\
\hline \multicolumn{11}{|c|}{$\begin{array}{l}\text { Fig. } 3 \text { a Overall association between thyroid autoimmunity and clinical miscarriage rate. Results showed no significant difference in clinical } \\
\left.\text { miscarriage rate between TAl+ and TAl- women overall (OR } 1.04 ; 95 \% \mathrm{Cl}[0.52,2.07] ; \mathrm{P}=0.908 ; 8 \text { studies; } \mathrm{I}^{2}=53 \%\right) \text {. b) Association between thyroid } \\
\text { autoimmunity and clinical miscarriage rate in euthyroid women. Results showed no significant difference in clinical miscarriage rate between } \\
\left.\text { TAl+ and TAl- euthyroid women (OR 1.18; } 95 \% \mathrm{Cl}[0.52,2.64] ; \mathrm{P}=0.69 ; 7 \text { studies; } I^{2}=54 \%\right) \text {. TAl+, thyroid autoimmune; TAl-, not thyroid } \\
\text { autoimmune; OR, odds ratio; } \mathrm{Cl} \text {, confidence interval }\end{array}$} \\
\hline
\end{tabular}

since patients in this study had a median age below 37 without any significant difference between TAI+ and TAI- groups [15], data from this study was included in other analyses. Analysis of the 13 remaining studies revealed no significant differences in patient age between all TAI+ and TAI- women (SMD 0.12; 95\%CI [- 0.04, 0.28 ]; $P=0.15 ; 13$ studies; $\mathrm{I}^{2}=76 \%$ ) (Fig. 10a). There were also no significant age differences between the sub- group of TAI+ and TAI- women who were euthyroid (SMD 0.13; 95\%CI $[-0.10,0.36] ; P=0.25 ; 10$ studies; $\left.\mathrm{I}^{2}=82 \%\right)$ (Fig. 10b).

\section{TSH}

TSH levels were reported by 6 studies and no significant difference was seen between TAI+ and TAI- women (SMD 0.28; 95\%CI $[-0.03,0.59] ; P=0.08 ; 6$ studies; $\mathrm{I}^{2}=$

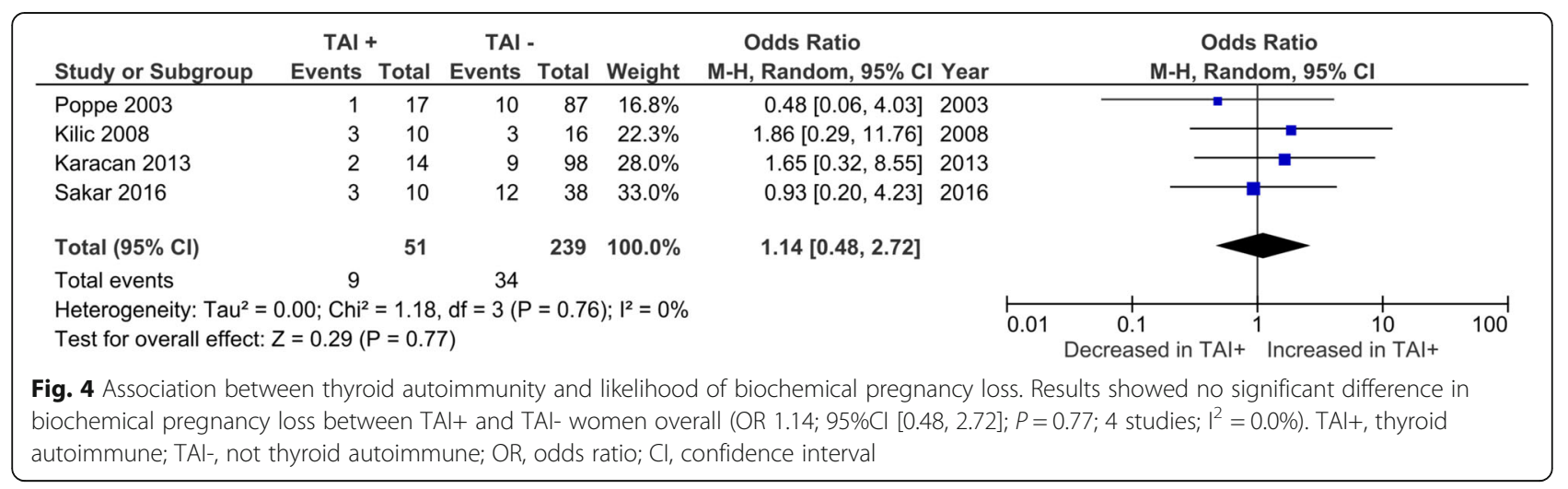




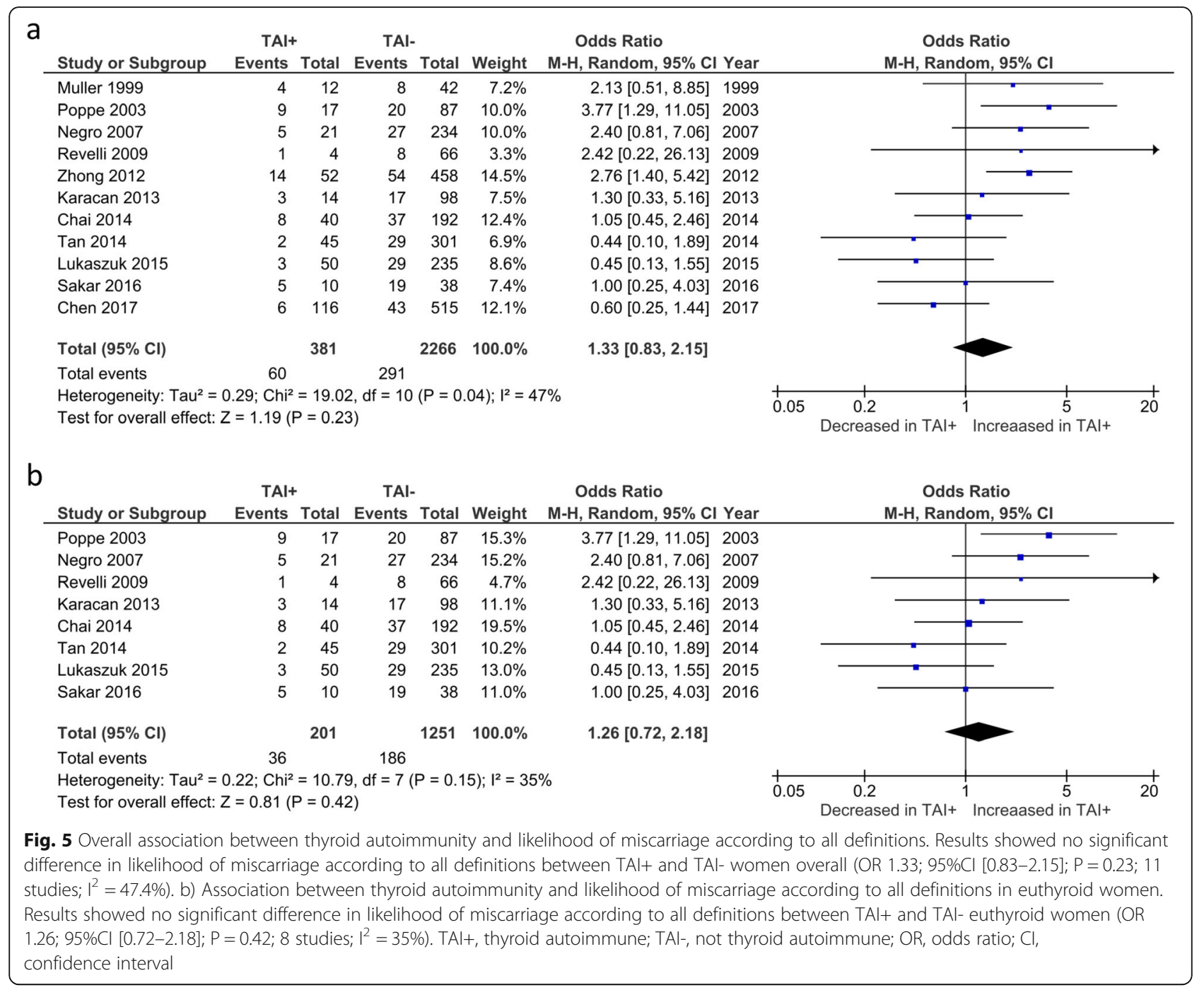

79\%)(Fig. 11a). In the 5 studies that reported TSH levels specifically in euthyroid women, no significant difference in TSH levels was seen between TAI+ and TAI- women (SMD 0.14; 95\%CI $[-0.07,0.34] ; P=0.19 ; 5$ studies; $\mathrm{I}^{2}=$ 46\%)(Fig. 11b).

\section{Heterogeneity and sensitivity analysis}

Analyses displaying moderate/high heterogeneity $\left(\mathrm{I}^{2} \geq\right.$ $50 \%$ ) were adjusted following sensitivity analysis (Table 2). Removal of 1-2 studies was sufficient to reduce heterogeneity in all cases. Following sensitivity

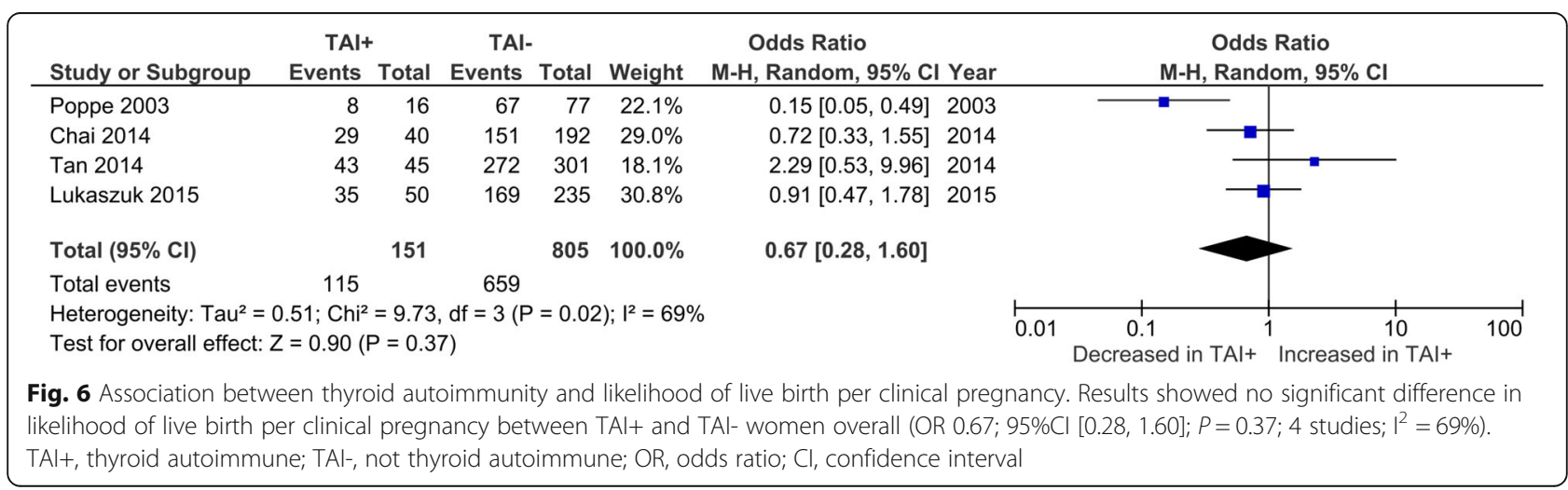




\begin{tabular}{|c|c|c|c|c|c|c|c|}
\hline Study or Subgroup & $\begin{array}{r}\text { TAl+ } \\
\text { Events }\end{array}$ & Total & $\begin{array}{l}\text { TAI- } \\
\text { Events }\end{array}$ & Total & Weight & $\begin{array}{l}\text { Odds Ratio } \\
\text { M-H, Random, } 95 \% \mathrm{Cl} \text { Year }\end{array}$ & $\begin{array}{c}\text { Odds Ratio } \\
\text { M-H, Random, } 95 \% \mathrm{Cl}\end{array}$ \\
\hline Poppe 2003 & 8 & 32 & 67 & 202 & $7.3 \%$ & $0.67[0.29,1.57] 2003$ & $\cdot$ \\
\hline Negro 2007 & 16 & 42 & 207 & 374 & $12.4 \%$ & $0.50[0.26,0.96] 2007$ & \\
\hline Tan 2014 & 43 & 110 & 272 & 725 & $30.8 \%$ & $1.07[0.71,1.61] 2014$ & \\
\hline Chai 2014 & 29 & 89 & 151 & 419 & $22.3 \%$ & $0.86[0.53,1.39] 2014$ & \\
\hline Lukaszuk 2015 & 35 & 114 & 169 & 495 & $27.2 \%$ & $0.85[0.55,1.33] 2015$ & \\
\hline Total $(95 \% \mathrm{Cl})$ & & 387 & & 2215 & $100.0 \%$ & $0.84[0.67,1.06]$ & 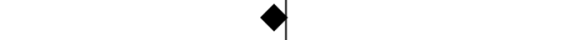 \\
\hline Total events & 131 & & 866 & & & & \\
\hline \multicolumn{7}{|c|}{$\begin{array}{l}\text { Heterogeneity: } \mathrm{Tau}^{2}=0.00 ; \mathrm{Chi}^{2}=4.07, \mathrm{df}=4(\mathrm{P}=0.40) ; \mathrm{I}^{2}=2 \% \\
\text { Test for overall effect: } Z=1.46(\mathrm{P}=0.15)\end{array}$} & $\begin{array}{|ccccc|}0.01 & 0.1 & 1 & 1 & 1 \\
& \text { Decreased in TAl+ } & & 1 & 1 \\
& \text { Increased in TAl+ } & & \end{array}$ \\
\hline
\end{tabular}

analyses, CPR, CMR, MR, LBR per CP and ET showed no change in statistical significance outcomes compared with unadjusted analyses. In NOR analyses, exclusion of Karacan et al. (2013) [34] led to significantly fewer oocytes being retrieved amongst TAI+ women (SMD -0.13; 95\%CI $[-0.22,-0.04], P=0.007)$.

\section{Publication Bias}

Results of Egger's weighted regression test indicated no publication bias in mean maternal age, CPR, overall MR or OPR.

\section{Quality analysis}

Of the 14 studies included, the quality ranged from 7 to 8 (Table 1). No studies were excluded from the analysis based on poor quality.

\section{Discussion}

Here we undertake the most comprehensive metaanalysis to-date regarding the effect of TAI on IVF/ICSI outcomes in women of known ages and TSH status. For the first time, we use strict criteria to analyse BPL and CMR as well as LBR on a per-cycle and per-CP basis in euthyroid women as well as in a larger group, which included women with $\mathrm{SCH}$. We found no difference in any pregnancy outcomes in relation to TAI status. Our results contrast with previous meta-analyses showing increased MR and/or decreased LBR in TAI+ women overall and in euthyroid women undergoing IVF/ICSI [6, $8,9]$. On the other hand, our findings are consistent with those of He et al. (2016) [10] and Leiva et al. (2017) [11]. There are important differences between our study and previous meta-analyses that may explain some of

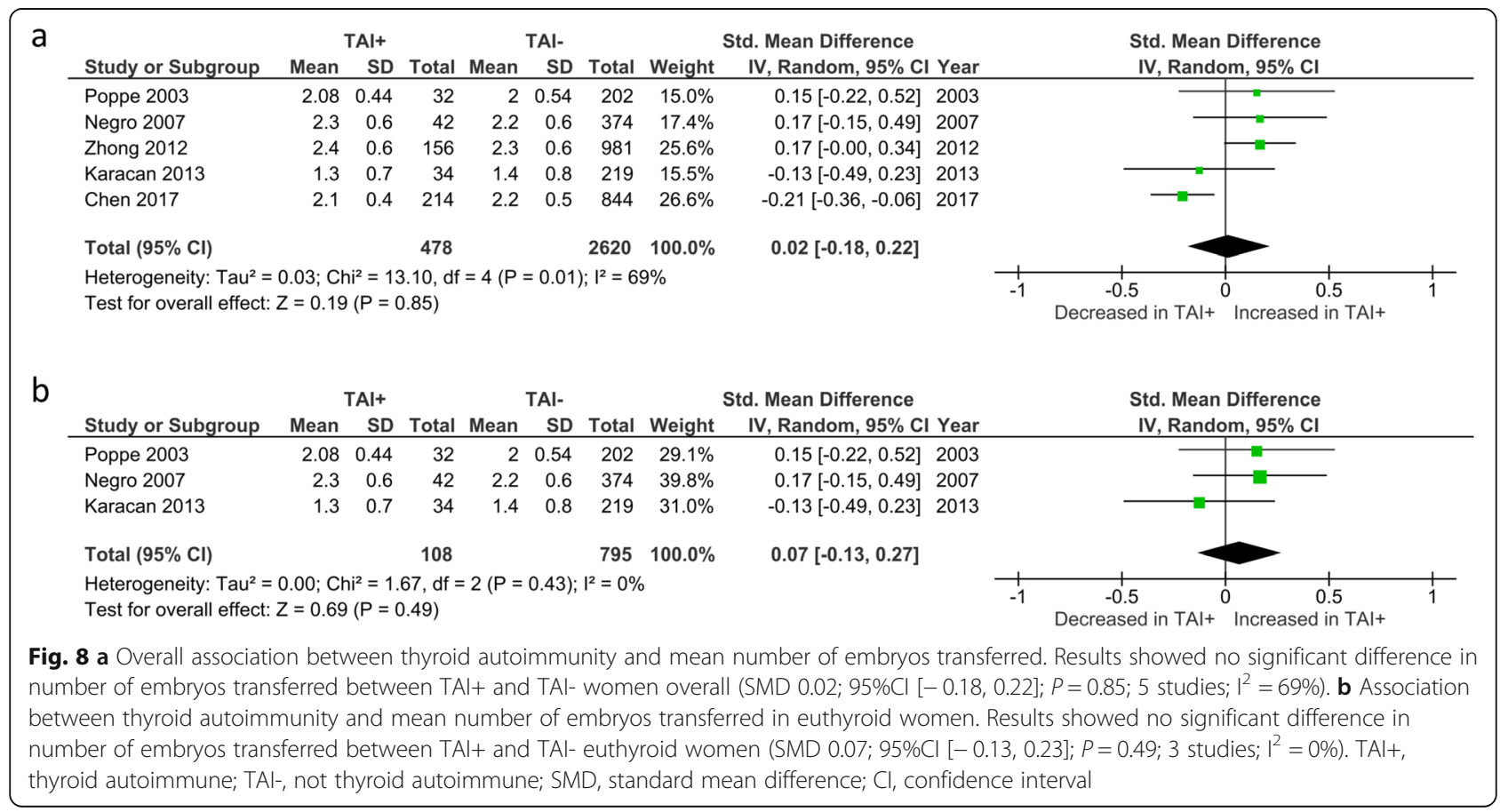




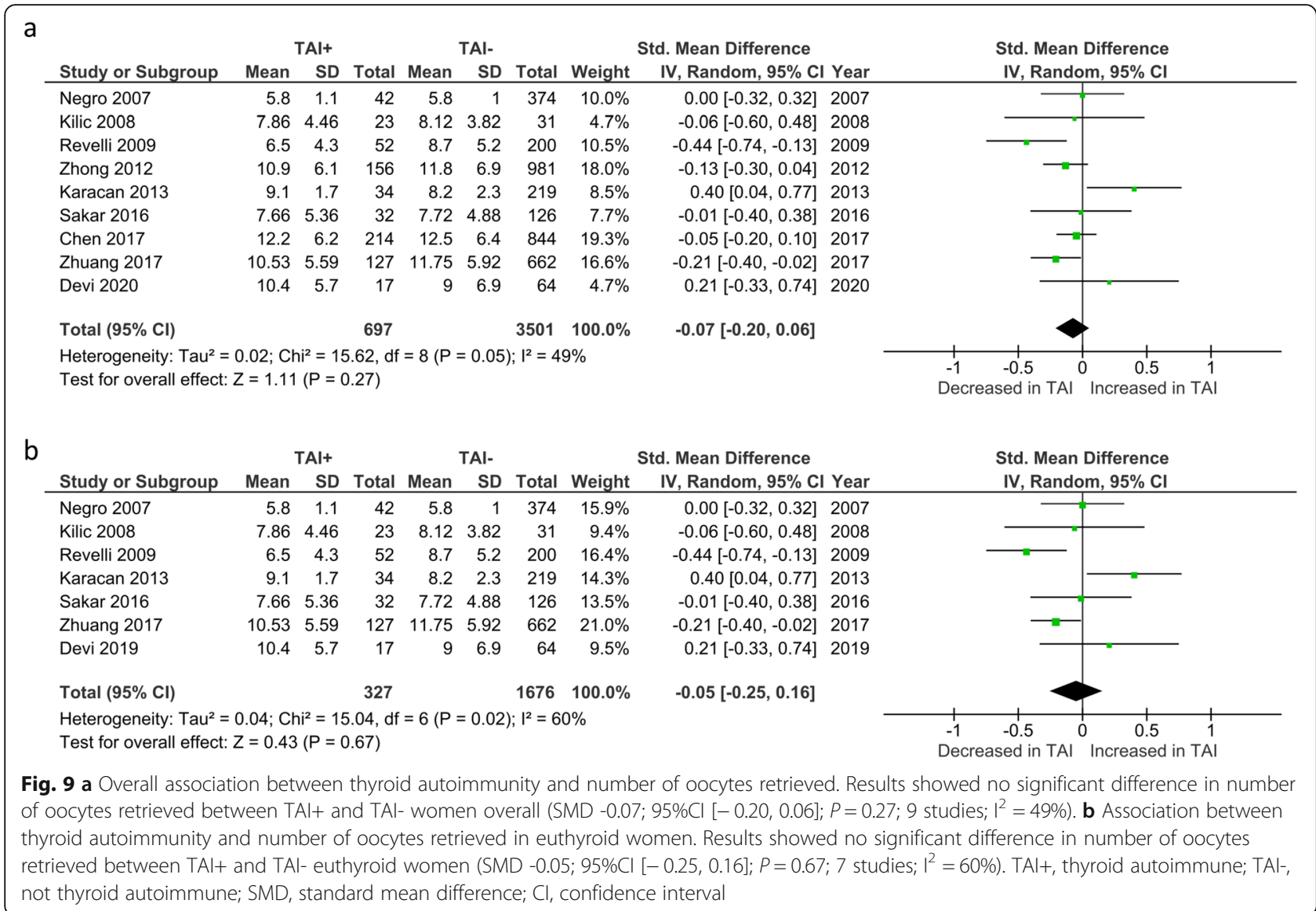

these discrepancies. Our systematic review included the most recent studies such as Devi et al. (2019) [22], Chen et al. (2017) [21] and Sakar et al. (2016) [18], which did not find any association between TAI positivity and pregnancy outcome. We also analysed outcomes in studies that were strictly limited to euthyroid women in addition to the wider group seen in previous studies. We were also strict in analysing like-for-like outcomes (e.g. CMR and LBR per cycle) rather than combining variously defined outcomes into a single analysis. Given the powerful influence of female age on pregnancy outcome through effects on oocyte quality [20], a critically important strength of our study was to restrict analyses to papers that reported female age. Importantly, we found that both groups were comparably aged thereby unequivocally ruling out the possibility of an age effect. In contrast, in the most recent meta-analysis, one quarter of included studies had not reported age and analysis of ages amongst those that did, found that TAI+ women were significantly older than TAI- women [6]. A metaregression analysis performed by the authors suggested that such differences were unlikely to have impacted their outcomes [6]. However, this cannot be concluded with certainty since, as the authors themselves noted, only 9 studies were included in the meta-regression, which is below the minimum of 10 recommended for avoiding Type II errors [29] added to which, ages were completely unknown for a substantial proportion of included studies. Furthermore, by considering maternal TSH levels, the present study accounted for potential confounding effects due to substantial thyroid dysfunction.

The lack of a detrimental effect of TAI on pregnancy outcomes that we find here is consistent with two recent large RCTs. The first RCT, the POSTAL study, tested whether levothyroxine supplementation could reduce $\mathrm{MR}$ in $600 \mathrm{TAI}+$ euthyroid women (mean age 31.6 years) undergoing IVF and found there was no benefit [36]. Since this study did not include a TAI- group, it is not known whether MR was actually increased in their untreated $\mathrm{TAI}+$ cohort. Indeed, the study was powered against a predicted CMR of $30 \%$ for $\mathrm{TAI}+$ women but unexpectedly, the actual MR in their untreated TAI+ arm was only $10.6 \%$, which was no higher, and indeed, somewhat lower than the background rate of $\sim 15 \%$ for women of a similar age $[18,19]$. The recently reported TABLET trial [37] studied a broader population of TAI+ women with a history of miscarriage or infertility and again found no benefit of levothyroxine supplementation. Significantly, however, the rate of combined CMR 


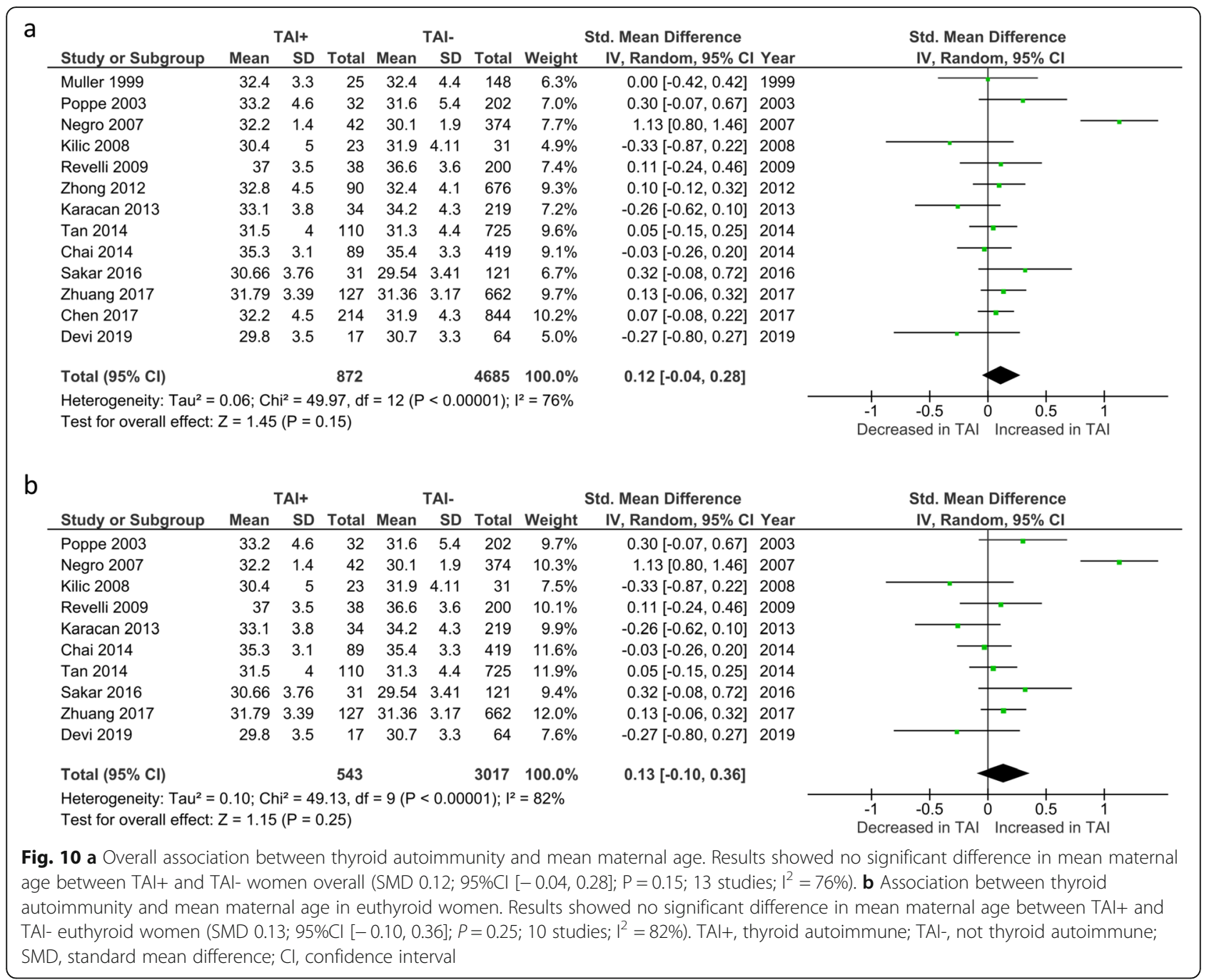

and BPL was $~ 30 \%$; this rate, which includes biochemical losses that occur with high frequency, also seems to be no higher than the predicted rate in the general population [16]. It is therefore likely that the lack of effect observed in both studies was at least in part because there isn't a markedly increased risk of miscarriage in the first place in TAI+ women, entirely consistent with our present findings.

There has been another recent meta-analysis that did not consider the full breadth of IVF patients as we did here, but instead focused only on studies in which ICSI was performed for fertilisation [38]. Consequently, it only contained 4 studies, 3 of which had been included in the earlier meta-analysis by Busnelli et al. (2016) [6], along with a more recent paper [23] which we included in our study. Significantly, Poppe et al. (2018) [38] found no difference in MR when analyses were strictly restricted to CPs, entirely in keeping with our findings. There have been a number of alternative theories regarding ICSI's ability to overcome the adverse effects of
TAI on pregnancy outcomes. It has been suggested that anti thyroid antibodies may bind antigens in the zona pellucida, thereby disrupting its function [39], and that ICSI may be able to overcome this effect. However, in light of the present study's findings, it appears more likely that the finding of Poppe et al. (2018) [38] are due to the inclusion of more recent studies and stricter inclusion criteria.

A recent meta-analysis investigating the effect of levothyroxine treatment found that MR in TAI+ women who conceived with ART were not reduced by levothyroxine but that levothyroxine decreased MR among TAI+ women who also had $\mathrm{SCH}$ [40] suggesting that $\mathrm{SCH}$ rather than TAI per se may confer the detrimental effect. Considering the recent change in guidelines, which now propose that $\mathrm{TAI}+$ women with $\mathrm{TSH}>2.5 \mathrm{mIU} / \mathrm{mL}$ should be supplemented with thyroxine [25, 41], most women with $\mathrm{SCH}$ and TAI were more likely to have been excluded from more recent studies. It is therefore possible that previous negative effects on pregnancy 


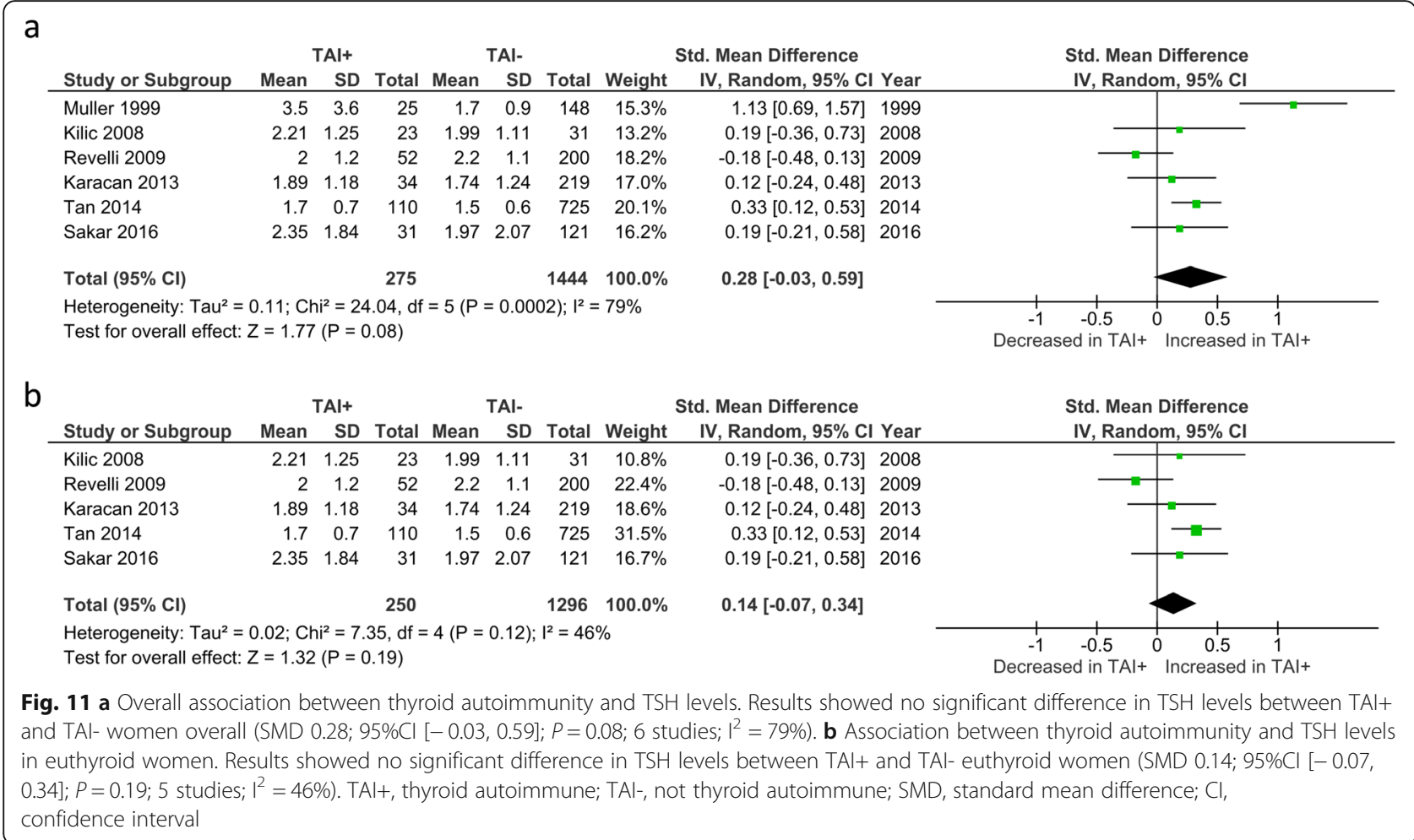

outcomes in TAI+ women may have been due to $\mathrm{SCH}$, rather than TAI itself. It is worth noting that a number of studies included in previous analyses that were not included in the present study did not report serum TSH levels, making it possible that the relationship between TAI and adverse pregnancy outcomes may have been due to thyroid dysfunction. We note, however, that the results of the present study do not support this notion since we found no association between TAI and pregnancy outcome either in euthyroid women or the overall group. Studies such as Chai et al. (2014) [14] and Zhuang et al. (2017) [24] separated their investigation on TAI and IVF based on maternal serum TSH levels $<2.5$ and $>2.5$ and found no difference in IVF outcomes based on TSH status. Furthermore, A recent retrospective study and meta-analysis found no increased risk of adverse pregnancy outcomes in women with $\mathrm{TSH}>2.5$ [42]. Thus, whilst this idea may hold some merit, further investigation is required.

We acknowledge that a variety of factors may influence the strength of our findings. Individual studies assessed different antibodies (TPO-Ab and/or TG-Ab) and used different assays as well as different cut-off values for defining TAI positivity (Table 1), which could potentially affect outcomes. Cause of infertility maybe be a potential confounding factor. A number of the included studies reported no significant difference in cause of infertility between the TAI+ and TAI- groups. However, each had varying inclusion criteria, and in recent a recent meta-analysis, TAI has been shown to be more prevalent in conditions associated with poor IVF outcomes such as PCOS [43]. It has been shown that the presence of TPO-Ab in particular is associated with an increased risk of PCOS and endometriosis, and varying aetiologies based on the inclusion criteria and antibodies present may have influenced results [36]. There were also limited details on individual patients such as stimulation protocols used, which could theoretically affect results given that antagonist and agonist protocols could have different effects on thyroid function [44]. The numbers of embryos transferred could also impact pregnancy outcomes. Although we considered this potential confounder, and analysis of the five studies that reported embryo numbers transferred showed no difference in relation to TAI status (Fig. 8), we did not have data on embryo numbers for 9 studies. We note as well that although we adhered to uniform criteria for outcomes, the included studies defined and reported clinical outcomes differently and some studies were excluded based on discrepancies in the reported data, a lack of clarity regarding definitions, or insufficient available data regarding clinical outcomes. It therefore remains possible that 
Table 2 Results of meta-analysis before and after sensitivity analysis

\begin{tabular}{|c|c|c|c|c|c|c|c|}
\hline Outcome & $\begin{array}{l}\text { Unadjusted OR / } \\
\text { SMD }(95 \% \mathrm{CI})\end{array}$ & $\begin{array}{l}P \\
\text { value }\end{array}$ & $1^{2}$ & $\begin{array}{l}\text { Studies Excluded Following } \\
\text { Sensitivity Analysis }\end{array}$ & $\begin{array}{l}\text { OR }(95 \% \mathrm{Cl}) \text { Following Exclusion of Studies } \\
\text { due to Sensitivity Analysis }\end{array}$ & $\begin{array}{l}P \\
\text { value } \\
\end{array}$ & $1^{2}$ \\
\hline$C P R$ - overall & $\begin{array}{l}\text { OR 0.86; 95\%Cl [0.70, } \\
1.05]\end{array}$ & 0.14 & $29 \%$ & & & & \\
\hline $\begin{array}{l}\text { CPR - } \\
\text { euthyroid }\end{array}$ & $\begin{array}{l}\text { OR 0.88; } 95 \% \text { Cl [0.69, } \\
1.12]\end{array}$ & 0.29 & $32 \%$ & & & & \\
\hline $\begin{array}{l}\text { CMR - } \\
\text { overall }\end{array}$ & $\begin{array}{l}\text { OR 1.04; } 95 \% \mathrm{Cl}[0.52, \\
2.07]\end{array}$ & 0.908 & $53 \%$ & Poppe 2003 & OR 0.74; 95\%Cl $[0.46,1.19)$ & 0.21 & $0.00 \%$ \\
\hline $\begin{array}{l}\text { CMR - } \\
\text { euthyroid }\end{array}$ & $\begin{array}{l}\text { OR 1.18; } 95 \% \mathrm{Cl}[0.52, \\
2.64]\end{array}$ & 0.69 & $54 \%$ & Poppe 2003 & OR $0.81 ; 95 \% \mathrm{Cl}[0.46,1.41]$ & 0.45 & $0.00 \%$ \\
\hline$B P L-$ overall & $\begin{array}{l}\text { OR 1.14; } 95 \% \mathrm{Cl}[0.48, \\
2.72]\end{array}$ & 0.769 & $0 \%$ & & & & \\
\hline $\begin{array}{l}\text { BPL- } \\
\text { euthyroid }\end{array}$ & - & & & & & & \\
\hline MR - overall & $\begin{array}{l}\text { OR 1.33; 95\%Cl [0.83, } \\
2.15]\end{array}$ & 0.23 & $47 \%$ & & & & \\
\hline $\begin{array}{l}\text { MR - } \\
\text { euthyroid }\end{array}$ & $\begin{array}{l}\text { OR } 1.26 ; 95 \% \mathrm{Cl} \\
{[0.72-2.18]}\end{array}$ & 0.42 & $35 \%$ & & & & \\
\hline $\begin{array}{l}\text { LBR/CP - } \\
\text { overall }\end{array}$ & $\begin{array}{l}\text { OR } 0.67 ; 95 \% \mathrm{Cl}[0.28, \\
1.60]\end{array}$ & 0.37 & $69 \%$ & Poppe 2003 & OR 0.92; 95\%Cl $[0.57,1.48]$ & 0.722 & $0.00 \%$ \\
\hline $\begin{array}{l}\text { LBR/CP - } \\
\text { euthyroid }\end{array}$ & - & & & & & & \\
\hline $\begin{array}{l}\text { LBR/cycle - } \\
\text { overall }\end{array}$ & $\begin{array}{l}\text { OR 0.84; } 95 \% \mathrm{Cl}[0.67 \\
1.06]\end{array}$ & 0.15 & $2 \%$ & & & & \\
\hline $\begin{array}{l}\text { LBR/cycle - } \\
\text { euthyroid }\end{array}$ & - & & & & & & \\
\hline$E T$ - overall & $\begin{array}{l}\mathrm{SMD} 0.02 ; 95 \% \mathrm{Cl} \\
{[-0.18,0.22]}\end{array}$ & 0.85 & $69 \%$ & Chen 2017 & SMD 0.13; $95 \% \mathrm{Cl}[-0.00,0.26]$ & 0.054 & $0.00 \%$ \\
\hline $\begin{array}{l}E T \text { - } \\
\text { euthyroid }\end{array}$ & $\begin{array}{l}\text { SMD } 0.07 ; 95 \% \mathrm{Cl}[- \\
0.13,0.2]\end{array}$ & 0.49 & $0 \%$ & & & & \\
\hline $\begin{array}{l}\text { NOR - } \\
\text { overall }\end{array}$ & $\begin{array}{l}\text { SMD }-0.07 ; 95 \% C l \\
{[-0.20,0.06]}\end{array}$ & 0.27 & $49 \%$ & & & & \\
\hline $\begin{array}{l}\text { NOR - } \\
\text { euthyroid }\end{array}$ & $\begin{array}{l}\text { SMD }-0.05 ; 95 \% \mathrm{Cl} \\
{[-0.25,0.16]}\end{array}$ & 0.67 & $60 \%$ & Karacan 2013 & $\mathrm{SMD}-0.14 ; 95 \% \mathrm{Cl}[-0.30,0.02]$ & 0.08 & $26 \%$ \\
\hline Age - overall & $\begin{array}{l}\text { SMD } 0.12 ; 95 \% \mathrm{Cl} \\
{[-0.04,0.28]}\end{array}$ & 0.15 & $76 \%$ & & & & \\
\hline $\begin{array}{l}\text { Age - } \\
\text { euthyroid }\end{array}$ & $\begin{array}{l}\text { SMD } 0.13 ; 95 \% \mathrm{Cl} \\
{[-0.10,0.36]}\end{array}$ & 0.25 & $82 \%$ & & & & \\
\hline TSH - overall & $\begin{array}{l}\text { SMD 0.28; } 95 \% \mathrm{Cl} \\
{[-0.03,0.59]}\end{array}$ & 0.08 & $79 \%$ & & & & \\
\hline $\begin{array}{l}\text { TSH - } \\
\text { euthyroid }\end{array}$ & $\begin{array}{l}\text { SMD 0.14; } 95 \% \mathrm{Cl} \\
{[-0.07,0.34]}\end{array}$ & 0.19 & $46 \%$ & & & & \\
\hline
\end{tabular}

$\mathrm{CPR}$, clinical pregnancy rate; $\mathrm{CMR}$, clinical miscarriage rate; $\mathrm{BPL}$, biochemical pregnancy loss; $\mathrm{MR}$; miscarriage rate according to all definitions; $\mathrm{LBR} / \mathrm{CP}$, live birth rate per clinical pregnancy; LBR/ cycle, live birth rate per cycle; IR, implantation rate; ET, mean number of embryos transferred; NOR, number of oocytes retrieved

some important data were omitted that may have influenced the results.

\section{Conclusions}

The results of the present study suggest that TAI has no effect on pregnancy outcomes in women undergoing IVF either overall, or in euthyroid women. The findings of this study may be useful when deciding on treatment plans for TAI+ women undergoing IVF.

\section{Supplementary Information}

The online version contains supplementary material available at https://doi. org/10.1186/s12958-020-00671-3.

\section{Additional file 1}

\section{Abbreviations}

TAl: Thyroid Autoimmunity; TPO-Ab: Anti-thyroid peroxidase antibodies; TGAb: Anti-thyroglobulin antibodies; MR: Miscarriage rate; CMR: Clinical miscarriage rate; BPL: Biochemical pregnancy loss; CP: Clinical pregnancy; CPR: Clinical pregnancy rate; LBR: Live birth rate; IR: Implantation rate; 
NOR: Number of oocytes retrieved; ET: Embryos transferred; TSH: Thyroid stimulating hormone; SCH: Subclinical hypothyroidism; OR: Odds ratio; $\mathrm{Cl}$ : Confidence interval

\section{Acknowledgements}

The authors acknowledge and thank Professor Mieke van Driel from the University of Queensland for her help in designing this review.

\section{Authors' contributions}

AV was involved in study design and undertook the majority of database searches, quality assessment, study selection, data extraction, data analysis, manuscript drafting, and critical discussion. WW assisted with database searches, quality assessment and data extraction. MW undertook statistical analyses, interpreting data and manuscript drafting. $\mathrm{HH}$ conceived and designed the project, assisted with data interpretation, manuscript drafting, manuscript revision and critical discussion. All authors read and approved the final manuscript.

\section{Funding}

This work was supported by the Christopher Chen Endowment and grants from the National Health and Medical Research Council (APP1078134, APP1103689 \& APP1122484) to HAH.

\section{Availability of data and materials}

The current study was based on results of relevant published studies.

\section{Ethics approval and consent to participate}

Not applicable.

\section{Consent for publication}

Not applicable.

\section{Competing interests}

The authors declare that they have no competing interests.

\section{Author details}

"Faculty of Medicine, The University of Queensland, Brisbane, Queensland, Australia. ${ }^{2}$ Faculty of Medicine, The University of Queensland, Brisbane, Queensland, Australia. ${ }^{3}$ QIMR Berghofer Medical Research Institute, Herston, Queensland, Australia. ${ }^{4}$ Christopher Chen Oocyte Biology Research Laboratory, UQ Centre for Clinical Research, The University of Queensland, Brisbane, Queensland, Australia. ${ }^{5}$ Reproductive Endocrinology and Infertility Clinic, Royal Brisbane \& Women's Hospital, Brisbane, Queensland, Australia. ${ }^{6}$ Queensland Fertility Group, Brisbane, Queensland, Australia.

\section{Received: 27 June 2020 Accepted: 8 November 2020}

\section{Published online: 25 November 2020}

\section{References}

1. Krassas GE, Poppe K, Glinoer D. Thyroid function and human reproductive health. Endocr Rev. 2010;31(5):702-55.

2. Vissenberg R, Manders VD, Mastenbroek S, Fliers E, Afink GB, Ris-Stalpers C, et al. Pathophysiological aspects of thyroid hormone disorders/thyroid peroxidase autoantibodies and reproduction. Hum Reprod Update. 2015; 21(3):378-87.

3. Ghazeeri GS, Kutteh WH. Autoimmune factors in reproductive failure. Curr Opin Obstet Gynecol. 2001;13(3):287-91.

4. Korevaar TIM, Derakhshan A, Taylor PN, Meima M, Chen L, Bliddal S, et al. Association of Thyroid Function Test Abnormalities and Thyroid Autoimmunity with Preterm Birth: a systematic review and meta-analysis. Jama. 2019;322(7):632-41.

5. van den Boogaard E, Vissenberg R, Land JA, van Wely M, van der Post JA, Goddijn M, et al. Significance of (sub) clinical thyroid dysfunction and thyroid autoimmunity before conception and in early pregnancy: a systematic review. Hum Reprod Update. 2011;17(5):605-19.

6. Busnelli A, Paffoni A, Fedele L, Somigliana E. The impact of thyroid autoimmunity on IVF/ICSI outcome: a systematic review and meta-analysis. Hum Reprod Update. 2016;22(6):775-90.

7. Poppe K, Glinoer D, Tournaye H, Schiettecatte J, Devroey P, van Steirteghem A, et al. Impact of ovarian hyperstimulation on thyroid function in women with and without thyroid autoimmunity. J Clin Endocrinol Metab. 2004; 89(8):3808-12.

8. Toulis KA, Goulis DG, Venetis CA, Kolibianakis EM, Negro R, Tarlatzis BC, et al. Risk of spontaneous miscarriage in euthyroid women with thyroid autoimmunity undergoing IVF: a meta-analysis. Eur J Endocrinol. 2010; 162(4):643-52

9. Thangaratinam S, Tan A, Knox E, Kilby MD, Franklyn J, Coomarasamy A. Association between thyroid autoantibodies and miscarriage and preterm birth: meta-analysis of evidence. Bmj. 2011;342:d2616.

10. He H, Jing S, Gong F, Tan YQ, Lu GX, Lin G. Effect of thyroid autoimmunity per se on assisted reproduction treatment outcomes: a meta-analysis. Taiwan J Obstet Gynecol. 2016;55(2):159-65.

11. Leiva P, Schwarze JE, Vasquez P, Ortega C, Villa S, Crosby J, et al. There is no association between the presence of anti-thyroid antibodies and increased reproductive loss in pregnant women after ART: a systematic review and meta-analysis. JBRA Assist Reprod. 2017;21(4):361-5.

12. Muller AF, Verhoeff A, Mantel MJ, Berghout A. Thyroid autoimmunity and abortion: a prospective study in women undergoing in vitro fertilization. Fertil Steril. 1999;71(1):30-4.

13. Poppe K, Glinoer D, Tournaye H, Devroey P, van Steirteghem A, Kaufman L, et al. Assisted reproduction and thyroid autoimmunity: an unfortunate combination? J Clin Endocrinol Metab. 2003;88(9):4149-52.

14. Chai J, Yeung WY, Lee CY, Li HW, Ho PC, Ng HY. Live birth rates following in vitro fertilization in women with thyroid autoimmunity and/or subclinical hypothyroidism. Clin Endocrinol. 2014;80(1):122-7.

15. Łukaszuk K, Kunicki M, Kulwikowska P, Liss J, Pastuszek E, Jaszczołt M, et al. The impact of the presence of antithyroid antibodies on pregnancy outcome following intracytoplasmatic sperm injection-ICSI and embryo transfer in women with normal thyreotropine levels. J Endocrinol Investig. 2015;38(12):1335-43.

16. Macklon NS, Geraedts JP, Fauser BC. Conception to ongoing pregnancy: the 'black box' of early pregnancy loss. Hum Reprod Update. 2002;8(4):333-43.

17. Zeadna A, Son WY, Moon JH, Dahan MH. A comparison of biochemical pregnancy rates between women who underwent IVF and fertile controls who conceived spontaneouslyt. Hum Reprod. 2015;30(4):783-8.

18. Saravelos SH, Li TC. Unexplained recurrent miscarriage: how can we explain it? Hum Reprod. 2012;27(7):1882-6.

19. Nybo Andersen AM, Wohlfahrt J, Christens P, Olsen J, Melbye M. Maternal age and fetal loss: population based register linkage study. Bmj. 2000; 320(7251):1708-12.

20. Greaney J, Wei Z, Homer H. Regulation of chromosome segregation in oocytes and the cellular basis for female meiotic errors. Hum Reprod Update. 2018;24(2):135-61.

21. Chen $X, M o ~ M L$, Huang CY, Diao LH, Li GG, Li YY, et al. Association of serum autoantibodies with pregnancy outcome of patients undergoing first IVF/ ICSI treatment: a prospective cohort study. J Reprod Immunol. 2017;122:1420.

22. Devi AB, Prasad SS, Koner BC. Antithyroid Antibodies and Fertility Outcome in Euthyroid Women Undergoing In Vitro Fertilisation. J Clin Diagnostic Res. 2019;13(5):QC05-08.

23. Sakar MN, Unal A, Atay AE, Zebitay AG, Verit FF, Demir S, et al. Is there an effect of thyroid autoimmunity on the outcomes of assisted reproduction? J Obstet Gynaecol. 2016;36(2):213-7.

24. Zhuang LL, Hao CF, Zhang N, Liu XY, Ge L, Ma JL. Effects of thyroidstimulating hormone and thyroid autoantibodies on pregnancy outcome after in vitro fertilization and embryo transfer. J Reprod Med. 2017:62:391-6.

25. Alexander EK, Pearce EN, Brent GA, Brown RS, Chen H, Dosiou C, et al. 2017 guidelines of the American Thyroid Association for the diagnosis and Management of Thyroid Disease during Pregnancy and the postpartum. Thyroid. 2017;27(3):315-89.

26. Kutteh WH, Yetman DL, Carr AC, Beck LA, Scott RT Jr. Increased prevalence of antithyroid antibodies identified in women with recurrent pregnancy loss but not in women undergoing assisted reproduction. Fertil Steril. 1999;71(5): 843-8.

27. Wells GA, Shea, B., O'Connell, D., Peterson, J., Welch, V., Tugwell, P. The Newcastle-Ottawa Scale (NOS) for assessing the quality of nonrandomized studies in metaanalyses.3rd Symposium on Systematic Reviews: Beyond the Basics 2000 [http://www.ohri.ca/programs/clinical_epidemiology/oxford.htm.].

28. StataCorp. Stata Statistical Software: Release 15. College Station: StataCorp LLC; 2017. 
29. Higgins JPT, Green S (eds). Cochrane Handbook for Systematic Reviews of Interventions Version 5.1.0 [updated March 2011]. The Cochrane Collaboration, 2011, published as a textbook by Wiley-Blackwell in September 2008. Available from www.cochrane-handbook.org.

30. Negro R, Formoso G, Coppola L, Presicce G, Mangieri T, Pezzarossa A, et al. Euthyroid women with autoimmune disease undergoing assisted reproduction technologies: the role of autoimmunity and thyroid function. $J$ Endocrinol Investig. 2007;30(1):3-8.

31. Kilic S, Tasdemir N, Yilmaz N, Yuksel B, Gul A, Batioglu S. The effect of antithyroid antibodies on endometrial volume, embryo grade and IVF outcome. Gynecol Endocrinol. 2008;24(11):649-55.

32. Revelli A, Casano S, Piane LD, Grassi G, Gennarelli G, Guidetti D, et al. A retrospective study on IVF outcome in euthyroid patients with anti-thyroid antibodies: effects of levothyroxine, acetyl-salicylic acid and prednisolone adjuvant treatments. Reprod Biol Endocrinol. 2009;7:137.

33. Zhong YP, Ying Y, Wu HT, Zhou CQ, Xu YW, Wang Q, et al. Relationship between antithyroid antibody and pregnancy outcome following in vitro fertilization and embryo transfer. Int J Med Sci. 2012;9(2):121-5.

34. Karacan M, Alwaeely F, Cebi Z, Berberoglugil M, Batukan M, Ulug M, et al. Effect of antithyroid antibodies on ICSI outcome in antiphospholipid antibody-negative euthyroid women. Reprod BioMed Online. 2013;27(4): 376-80.

35. Tan S, Dieterle S, Pechlavanis S, Janssen OE, Fuhrer D. Thyroid autoantibodies per se do not impair intracytoplasmic sperm injection outcome in euthyroid healthy women. Eur J Endocrinol. 2014;170(4):495500.

36. Wang H, Gao H, Chi H, Zeng L, Xiao W, Wang Y, et al. Effect of levothyroxine on miscarriage among women with Normal thyroid function and thyroid autoimmunity undergoing in vitro fertilization and embryo transfer: a randomized clinical trial. Jama. 2017;318(22):2190-8.

37. Dhillon-Smith RK, Middleton L, Sunner KK, Cheed V, Baker K, Farrell-Carver $\mathrm{S}$, et al. Levothyroxine in women with thyroid peroxidase antibodies before conception. N Engl J Med. 2019;380(14):1316-25.

38. Poppe K, Autin C, Veltri F, Kleynen P, Grabczan L, Rozenberg S, et al. Thyroid autoimmunity and intracytoplasmic sperm injection outcome: a systematic review and meta-analysis. J Clin Endocrinol Metab. 2018;103(5):1755-66.

39. Monteleone P, Parrini D, Faviana P. Carletti E, Casarosa E, Uccelli A, et al. Female infertility related to thyroid autoimmunity: the ovarian follicle hypothesis. Am J Reprod Immunol. 2011;66(2):108-14.

40. Rao M, Zeng Z, Zhou F, Wang H, Liu J, Wang R, et al. Effect of levothyroxine supplementation on pregnancy loss and preterm birth in women with subclinical hypothyroidism and thyroid autoimmunity: a systematic review and meta-analysis. Hum Reprod Update. 2019;25(3):344-61.

41. Practice Committee of the American Society for Reproductive Medicine. Subclinical hypothyroidism in the infertile female population: a guideline. Fertil Steril. 2015;104(3):545-53.

42. Jin L, Wang M, Yue J, Zhu G-J, Zhang B. Association between TSH level and pregnancy outcomes in Euthyroid women undergoing IVF/ICSI: a retrospective study and meta-analysis. Curr Med Sci. 2019;39(4):631-7.

43. Romitti M, Fabris VC, Ziegelmann PK, Maia AL, Spritzer PM. Association between PCOS and autoimmune thyroid disease: a systematic review and meta-analysis. Endocr Connect. 2018;7(11):1158-67.

44. Gizzo S, Noventa M, Quaranta M, Vitagliano A, Esposito F, Andrisani A, et al. The potential role of GnRH agonists and antagonists in inducing thyroid Physiopathological changes during IVF. Reprod Sci. 2016;23(4):515-23.

\section{Publisher's Note}

Springer Nature remains neutral with regard to jurisdictional claims in published maps and institutional affiliations.

Ready to submit your research? Choose BMC and benefit from:

- fast, convenient online submission

- thorough peer review by experienced researchers in your field

- rapid publication on acceptance

- support for research data, including large and complex data types

- gold Open Access which fosters wider collaboration and increased citations

- maximum visibility for your research: over $100 \mathrm{M}$ website views per year

At BMC, research is always in progress.

Learn more biomedcentral.com/submissions 